\title{
Long-term impact of childhood malaria infection on school performance among school children in a malaria endemic area along the Thai-Myanmar border
}

Nutchavadee Vorasan, Wirichada Pan-Ngum, Podjanee Jittamala, Wanchai Maneeboonyang, Prasert Rukmanee and Saranath Lawpoolsri

\begin{abstract}
Background: Children represent a high-risk group for malaria worldwide. Among people in Thailand who have malaria during childhood, some may have multiple malaria attacks during their lifetime. Malaria may affect neurological cognition in children, resulting in short-term impairment of memory and language functions. However, little is known regarding the long-term effects of malaria infection on cognitive function. This study examines the long-term impact of malaria infection on school performance among school children living in a malaria-endemic area along the Thai-Myanmar border.

Methods: A retrospective cohort study was conducted among school children aged 6-17 years in a primary-secondary school of a sub-district of Ratchaburi Province, Thailand. History of childhood malaria infection was obtained from the medical records of the sole malaria clinic in the area. School performance was assessed by using scores for the subjects Thai Language and Mathematics in 2014. Other variables, such as demographic characteristics, perinatal history, nutritional status, and emotional intelligence, were also documented.

Results: A total of 457 students were included, 135 (30\%) of whom had a history of uncomplicated malaria infection. About half of the malaria-infected children had suffered infection before the age of four years. The mean scores for both Mathematics and Thai Language decreased in relation to the increasing number of malaria attacks. Most students had their last malaria episode more than two years previously. The mean scores were not associated with duration since the last malaria attack. The association between malaria infection and school performance was not significant after adjusting for potential confounders, including gender, school absenteeism over a semester term, and emotional intelligence.
\end{abstract}

Conclusions: This study characterizes the long-term consequences of uncomplicated malaria disease during childhood. School performance was not associated with a history of malaria infection, considering that most students had their last malaria infection more than two years previously. These findings indicate that the impact of uncomplicated malaria infection on school performance may not be prolonged.

Keywords: Malaria, Cognitive, School performance, Nutritional status, Emotional intelligence

*Correspondence: saranath.law@mahidol.ac.th

Department of Tropical Hygiene, Faculty of Tropical Medicine, Mahidol

University, Bangkok, Thailand

C Biomed Central

(c) 2015 Vorasan et al. This article is distributed under the terms of the Creative Commons Attribution 4.0 International License (http://creativecommons.org/licenses/by/4.0/), which permits unrestricted use, distribution, and reproduction in any medium, provided you give appropriate credit to the original author(s) and the source, provide a link to the Creative Commons license, and indicate if changes were made. The Creative Commons Public Domain Dedication waiver (http://creativecommons.org/ publicdomain/zero/1.0/) applies to the data made available in this article, unless otherwise stated. 


\section{Background}

Malaria infection remains a major public health problem worldwide. The World Health Organization has estimated that malaria affects 198 million individuals, and globally 584,000 persons died from the disease in 2013 $[1,2]$. Children are at high risk of malaria infection and are more likely to have serious conditions once infected [1-4]. In addition, malaria may affect neurological and physical development in children [5-9], especially when infection occurs in young children [10-13]. Malaria infection may affect cognitive performance, which leads to impairment of memory and language functions [1013]. Previous studies suggested that childhood malaria infection is associated with poor school performance [14-18]. Malaria infection is an important cause of school absenteeism among African children, which may affect their school performance [3]. In African children, impaired cognitive function can persist up to two years after cerebral malaria episode [3]. Moreover, multiple attacks of uncomplicated malaria in childhood may also affect cognitive function and school performance [12]. However, most findings were based on cross-sectional or cohort studies of short duration (1-2 years), which only represent the short-term impact of malaria infection on cognitive functions in children [14-18]. In addition, data on long-term cognitive impairment after malaria infection are derived mainly from studies of children with severe malaria $[8,9]$. There is still a lack of evidence regarding the long-term impact of uncomplicated malaria infection on cognitive function among children in settings of low endemicity where a relatively large atrisk population resides [19].

Thailand is considered as a malaria-endemic country. Although the overall endemicity is low nationwide, the transmission is most intense in rural areas along the international borders. In these areas malaria remains the major cause of illness among children, many of whom may experience multiple malaria attacks during their childhood [20]. However, there is no available evidence regarding the impact of childhood malaria infection on the cognitive function of these children, especially over the long term. Therefore, this study aimed to determine the impact of malaria by means of a retrospective cohort study on school performance over more than 10 years among school children in a primary-secondary school located in an area of low malaria endemicity along the Thai-Myanmar border. The longitudinal data of the student cohort allow assessment of the long-term impact of malaria infection on cognitive function through analysis of school performance. Findings from this study should provide further insight into the consequences of childhood malaria infection among children living in areas of low malaria endemicity.

\section{Methods \\ Study area}

The study was conducted in Tanousri, Suanphuang, Ratchaburi Province, located on the western border of Thailand. This is a mountainous area bordering Myanmar, where malaria transmission persists throughout the year. The Rajanagarindra Tropical Diseases Center (RTIC) operated by Mahidol University, the sole malaria clinic in this area, has provided free diagnosis and treatment of malaria to people in this district for more than 15 years. The vast majority of malaria cases in the area are detected at this center. There is only one primary-secondary school in Tanousri, which provides free tuition and education to approximately 1000 students.

\section{Study population}

Children aged 6-17 years old who were studying in 2014 at the Rujirapat primary-secondary school of Tanousri, Suanphuang, Ratchaburi Province, Thailand, and were permanent residents in the area, were enrolled in the study. Students who had a history of meningitis, encephalitis, and other central nervous system infections were excluded.

\section{Study design}

A retrospective cohort study was conducted among students in the school. History of malaria infection since birth was obtained from the medical records of the RITC. All malaria cases were based on microscopic diagnosis by trained and experienced staff. The participating students were classified into malaria and non-malaria groups according to their history of malaria infection. History of intestinal parasitic infection was obtained from the most recent mass stool examination survey in 2013 performed by trained personnel. Information on school performance (outcome) and other variables such as demographic characteristics, perinatal and birth history, nutritional status, and emotional intelligence were primarily collected in 2014.

\section{Assessment of school performance}

School performance was assessed using pupils' scores in the subjects Mathematics and Thai Language, graded by class teachers and ranging from 0 to 100 . The education tests were routinely developed following a standard protocol of the school, which regularly received quality assurance by the Ministry of Education, Thailand. Students in the same grade were tested with the same set of paper examination. The level of school performance was classified according to a standardization protocol: poor school performance was identified when a z-score test was below zero [17]. This standardization technique 
helps to avoid bias that might occur due to variation in standards of grading by different teachers; different teachers may have different evaluation standards that may lead to variation in test scores of children in different classrooms.

\section{Assessment of nutritional status}

Weight and height of students were collected from school records, documented routinely by the school. The measurement was performed by trained teachers following standard procedures provided by the Ministry of Public Health. Nutritional status was assessed following the standard guidelines of WHO, including weight for age and height for age. Poor nutritional status was considered when weight for age and height for age was below -2.0 of the standardized z-score $[21,22]$.

\section{Assessment of emotional intelligence}

Emotional intelligence was assessed by a standard emotional intelligence test (EQ test) developed by the Ministry of Public Health of Thailand. This standard questionnaire for EQ screening was tested among children in different parts of Thailand, and shown to have high validity and reliability [23]. Following the instructions for the test, children aged 6-11 years were assessed by trained teachers and children aged 12-17 years were assessed by self-evaluation of students.

The outcome of the EQ test was classified according to the guidelines of the Ministry of Public Health of Thailand. Students were classified as having low emotional intelligence if the test score was below 40 out of 100 for students aged 6-11 years and below 140 out of 208 for those aged $12-17$ years $[24,25]$.

\section{Collection of demographic, socioeconomic, and perinatal history data}

Students and their parents were interviewed using a structured questionnaire. Questions concerned basic demographic characteristics and socioeconomic status. Perinatal and birth histories of students were obtained from the review of the perinatal log book (if available).

\section{Data analysis}

All analyses were performed using SPSS version 18.0 (SPSS Inc., Chicago, IL, USA). Significance was considered at an alpha level of less than $0.05(\mathrm{p}<0.05)$. The Student $\mathrm{t}$-test and analysis of variance were used to analyse mean differences between groups. Logistic regression was used to determine crude odds ratio (OR) and $95 \%$ confidence interval (CI) for predictive factors of school performance. Factors that showed significant associations were then included in multivariate analysis to estimate adjusted ORs and $95 \%$ CIs.

\section{Ethical considerations}

The study was approved by the Ethical Review Committee of the Faculty of Tropical Medicine, Mahidol University, Thailand. Informed consent and assent forms were obtained from parents and students who participated in the study.

\section{Results}

\section{General and socioeconomic characteristics}

A total of 457 school children were enrolled. About half of the students were male (52\%) and in the age group 6-11 years (53\%). The majority of students (82\%) were Karen, an ethnic minority that live along the Thai-Myanmar border. Most fathers and mothers of students were uneducated (60 and $70 \%$, respectively), and $67 \%$ of students were from low-income families $(<30,000$ baht per year). More than $70 \%$ of students were term delivered with a birth weight of $2500 \mathrm{~g}$ or more (Table 1). Although almost all students had no complications at birth, some had a history of breech presentation and neonatal jaundice.

Most students had normal nutritional status in terms of weight for age and height for age. However, more than one-third (39\%) had low emotional intelligence. Most students attended the school regularly; only $19 \%$ accumulated absenteeism of seven days or more. A total of 202 students were tested for intestinal parasitic infection in 2013. Among them, 98 (49 \%) had been infected with intestinal parasites and subsequently received treatment (Table 1). The prevalence of parasitic infection was hookworm $24 \%$, Ascaris lumbricoides $8 \%$, Giardia lamblia $7 \%$, Trichuris trichiura $2 \%$, Enterobius vermicularis $2 \%$, and Opisthorchis viverrini $2 \%$ respectively. Approximately $30 \%$ of children had infected with more than one species of parasite.

A history of childhood malaria infection was recorded in 135 students (30\%), all of whom had uncomplicated malaria. The majority of malaria cases were infected with either Plasmodium falciparum or Plasmodium vivax. Only two students were infected with Plasmodium malariae. Among these children, 99 (73 \%) had experienced 1-2 malaria attacks and 13 (17 \%) had had five or more malaria attacks since birth. Almost half of the malaria-infected students (45\%) had their first malaria infection during the first four years of life. Recent infection (within $<1$ year) was observed in only 4 students. For the majority of malaria-infected students $(80 \%)$ it had been three years or more since their last malaria infection (Table 2).

\section{Association between childhood malaria infection and school performance}

Mean scores for both Mathematics and Thai Language significantly decreased in relation to the increasing 
Table 1 General and socioeconomic characteristics of schoolchildren

\begin{tabular}{|c|c|c|}
\hline Variable & $\mathbf{N}$ & Percentage \\
\hline Total & 457 & \\
\hline \multicolumn{3}{|l|}{ Sex } \\
\hline Male & 237 & 51.9 \\
\hline Female & 220 & 48.1 \\
\hline \multicolumn{3}{|l|}{ Age (years) } \\
\hline $6-11$ & 244 & 53.4 \\
\hline $12-17$ & 213 & 46.6 \\
\hline \multicolumn{3}{|l|}{ Ethnicity } \\
\hline Karen & 374 & 81.8 \\
\hline Thai & 83 & 18.2 \\
\hline \multicolumn{3}{|l|}{ Perinatal history } \\
\hline \multicolumn{3}{|l|}{ Birth weight (grams) } \\
\hline$<2500$ & 123 & 28.4 \\
\hline$\geq 2500$ & 310 & 71.6 \\
\hline \multicolumn{3}{|l|}{ Gestational age } \\
\hline$<36$ weeks & 120 & 27.6 \\
\hline$\geq 36$ weeks & 315 & 72.4 \\
\hline \multicolumn{3}{|l|}{ Father's education } \\
\hline Uneducated & 272 & 59.5 \\
\hline Educated & 185 & 40.5 \\
\hline \multicolumn{3}{|l|}{ Mother's education } \\
\hline Uneducated & 323 & 70.7 \\
\hline Educated & 134 & 29.3 \\
\hline \multicolumn{3}{|c|}{ Family income per year (baht) } \\
\hline$<30,000$ & 306 & 67.0 \\
\hline$\geq 30,000$ & 151 & 33.0 \\
\hline \multicolumn{3}{|l|}{ History of malaria infection } \\
\hline Non-malaria infected & 322 & 70.5 \\
\hline Malaria infected & 135 & 29.5 \\
\hline \multicolumn{3}{|c|}{ Results of Intestinal parasitic infection (only those detected; $\mathrm{n}=202$ ) } \\
\hline No infection & 104 & 51.5 \\
\hline Infection & 98 & 48.5 \\
\hline \multicolumn{3}{|l|}{ Nutritional status } \\
\hline \multicolumn{3}{|l|}{ Weight for age } \\
\hline Normal & 406 & 88.8 \\
\hline Poor nutritional status & 51 & 11.2 \\
\hline \multicolumn{3}{|l|}{ Height for Age } \\
\hline Normal & 392 & 85.8 \\
\hline Poor nutritional status & 65 & 14.2 \\
\hline \multicolumn{3}{|c|}{ School absenteeism (during a semester term) } \\
\hline$<1$ week & 372 & 81.4 \\
\hline$\geq 1$ week & 85 & 18.6 \\
\hline \multicolumn{3}{|l|}{ Emotional intelligence } \\
\hline Good & 82 & 17.9 \\
\hline Moderate & 195 & 42.7 \\
\hline Low & 180 & 39.4 \\
\hline
\end{tabular}

Table 2 Pattern of malaria infection among malariainfected students

\begin{tabular}{lcc}
\hline Variable & N & Percentage \\
\hline Total & 135 & \\
Type of malaria infection & & \\
$\quad$ Plasmodium falciparum (PF) & 50 & 37.0 \\
Plasmodium vivax (PV) & 45 & 33.4 \\
Plasmodium malariae (PM) & 1 & 0.7 \\
Mixed infection (PF and PV) & 5 & 3.7 \\
PF + PV & 33 & 24.5 \\
PM + PV & 1 & 0.7 \\
Number of malaria attacks & & \\
$1-2$ & 99 & 73.3 \\
$3-4$ & 23 & 17.0 \\
$\geq 5$ & 13 & 9.6 \\
Age at first malaria infection (years) & & \\
$0-4$ & 61 & 45.2 \\
$>4$ & 74 & 54.8 \\
Duration since last malaria attack (years) & & \\
$<1$ & 4 & 3.0 \\
$1-2$ & 19 & 24.4 \\
$3-4$ & 33 & 58.5 \\
$\geq 5$ & 79 & \\
\hline
\end{tabular}

* Multiple episodes

number of malaria attacks (Table 3). Students who had a history of five or more malaria episodes had lower scores in Mathematics and Thai Language than those who had only one or two malaria episodes. However, the mean score in Mathematics and Thai Language showed no statistically significant difference regarding the length of time since the last malaria infection (Table 4).

Factors associated with school performance

School performance was identified according to the standardized score. Of 457 students, 211, 220, and 168 students had poor scores in Mathematics, Thai Language, and both, respectively. Factors associated with poor school performance were firstly identified separately for each Mathematics or Thai Language test. The results of each individual analysis showed similar patterns regarding the predictive factors. Therefore, only the results of school performance defined by both Mathematics and Thai Language tests are presented here. Overall, approximately $37 \%$ of the students $(n=168)$ had poor school performance in test scores of both Mathematics and Thai Language. A history of malaria infection, in terms of malaria infection, number of malaria attacks, age at 
Table 3 Mean and standard deviation of school performance by number of malaria attacks

\begin{tabular}{|c|c|c|c|c|c|}
\hline \multirow[t]{2}{*}{ Variable } & \multicolumn{4}{|c|}{ Number of malaria attacks } & \multirow[t]{2}{*}{$\mathrm{p}$} \\
\hline & $0(n=322)$ & $1-2(n=99)$ & $3-4(n=23)$ & $\geq 5(n=13)$ & \\
\hline \multicolumn{6}{|l|}{ School performance } \\
\hline Mathematics score & $72.1(13.7)$ & $70.0(12.9)$ & $65.5(20.6)$ & $57.6(26.7)$ & 0.001 \\
\hline Thai language score & $74.5(10.9)$ & $73.7(8.37)$ & $73.0(8.32)$ & $69.2(7.37)$ & 0.04 \\
\hline
\end{tabular}

Table 4 Mean and standard deviation of school performance by duration since the last malaria infection

\begin{tabular}{|c|c|c|c|c|c|}
\hline \multirow[t]{2}{*}{ Variable } & \multicolumn{4}{|c|}{ Duration since last malaria infection (years) } & \multirow[t]{2}{*}{$\mathrm{p}$} \\
\hline & $\begin{array}{l}<1 \\
(n=4)\end{array}$ & $\begin{array}{l}1-2 \\
(n=19)\end{array}$ & $\begin{array}{l}3-4 \\
(n=33)\end{array}$ & $\begin{array}{l}\geq 5 \\
(n=79)\end{array}$ & \\
\hline \multicolumn{6}{|c|}{ School performance } \\
\hline $\begin{array}{l}\text { Mathematics } \\
\text { score }\end{array}$ & $76.5(8.8)$ & $69.6(13.8)$ & $67.3(15.7)$ & $67.7(17.2)$ & 0.72 \\
\hline $\begin{array}{l}\text { Thai language } \\
\text { score }\end{array}$ & $73.5(10.1)$ & $72.2(6.8)$ & $72.5(9.45)$ & $73.8(8.26)$ & 0.84 \\
\hline
\end{tabular}

first malaria infection, and duration since the last attack, was not significantly associated with school performance, even after adjusting for potential confounders (Table 5).

Male students were more than three times more likely than female students to have poor school performance (adjusted OR 3.38, 95 \% CI 2.22-5.17). Poor school performance was also observed more among younger students (aged 6-11 years) than in those 12-17 years old. In addition, students who had more school absenteeism ( $\geq 1$ week) were about 1.7 times more likely to have poor school performance. Low emotional intelligence was also associated with poor school performance (adjusted OR 2.21, 95 \% CI 1.43-3.41). However, ethnicity of students, parents' education, family income, perinatal history, history of parasitic infection, and nutritional status were not significantly associated with poor school performance (Table 5).

\section{Discussion}

Children are considered as a high-risk group for malaria infection. There are concerns regarding the consequences of malaria infection in small children, since it may affect the neurodevelopment of infants. Previous studies have shown that malaria infection may affect cognitive function and lead to impairments to memory and language function [10-14]. These studies were mostly conducted in areas of high malaria transmission, where severe malaria among children is more common, or evaluated cognitive function after malaria infection over the short term (1-2 years). In the present study, the long-term effect of malaria infection on cognitive function, as indicated by school performance in mathematics and language, was assessed among children living in an area of low malaria transmission.

Although malaria transmission is considered low in this area, $30 \%$ of school children had experienced at least one malaria infection and about half had had a malaria infection during the first four years of life, the critical period for brain development [26, 27]. However, the results of the study showed that childhood malaria infection was not associated with poor school performance. In addition, duration since the last malaria infection was also not significantly associated with school performance, although those who had last malaria attack within two years were likely to have poor school performance (OR 1.91). Since it was longer than three years since the last malaria infection for the majority $(80 \%)$ of malariainfected children in this study, these findings may reflect the true long-term consequences of malaria infection. These results are inconsistent with those of other studies that demonstrated a short-term impact of malaria infection on school performance [14-18]. Although some previous studies have also reported a significant long-term impact of malaria on school performance [8, 9], these studies were conducted among children with severe malaria, whereas all malaria cases observed in this study were uncomplicated infection. This suggests that the long-term effects on cognitive function might depend on the severity of malaria infection.

Numerous school children in this study had undergone multiple malaria episodes since birth. The data showed that the mean test scores in both mathematics and language were significantly associated with the number of malaria attacks. These findings are consistent with those reported from a study conducted in Sri Lanka [14]. However, the effect of multiple malaria attacks was not observed when poor school performance was classified using a standardized score.

This study showed that males had a greater tendency than females to perform poorly in school. In general, girls are reported to have greater concentration levels during study than boys, perhaps because boys usually are more inclined towards playing or outdoor activities than learning in the 
Table 5 Associations between other factors with school performance in test scores of mathematics/Thai Language

\begin{tabular}{|c|c|c|c|c|}
\hline \multirow[t]{2}{*}{ Variable } & \multicolumn{2}{|c|}{$\begin{array}{l}\text { School performance in test score of mathematics/ } \\
\text { Thai Language }\end{array}$} & \multirow[t]{2}{*}{ Crude OR } & \multirow[t]{2}{*}{ Adjusted OR } \\
\hline & Poor $(n=168)$ & Not poor $(n=289)$ & & \\
\hline \multicolumn{5}{|c|}{ History of malaria infection } \\
\hline Non-malaria infected & $117(36.3)$ & $205(63.7)$ & 1.00 & 1.00 \\
\hline Malaria infected & $51(37.8)$ & $84(62.2)$ & $1.06(0.70-1.61)$ & $0.90(0.56-1.45)$ \\
\hline \multicolumn{5}{|c|}{ Number of malaria attacks } \\
\hline 0 & $117(36.3)$ & $205(63.7)$ & 1.00 & \\
\hline 1 & $25(26.8)$ & $43(63.2)$ & $1.02(0.59-1.75)$ & \\
\hline$>1$ & $168(26.8)$ & $41(61.2)$ & $1.11(0.65-1.91)$ & \\
\hline \multicolumn{5}{|c|}{ Age at first malaria infection } \\
\hline$>4$ years & $26(35.1)$ & $48(64.9)$ & 1.00 & \\
\hline $0-4$ years & $25(41.0)$ & $36(59.0)$ & $1.28(0.64-2.58)$ & \\
\hline \multicolumn{5}{|c|}{ Duration since last malaria attack } \\
\hline Non-malaria infected & $117(36.3)$ & $205(63.7)$ & 1.00 & \\
\hline$\leq 2$ years & $12(52.2)$ & $11(47.8)$ & $1.91(0.81-4.47)$ & \\
\hline $3-4$ years & $8(24.2)$ & $25(75.8)$ & $0.56(0.24-1.28)$ & \\
\hline$\geq 5$ years & $31(39.2)$ & $48(60.8)$ & $1.13(0.68-1.87)$ & \\
\hline \multicolumn{5}{|l|}{ Sex } \\
\hline Female & $48(21.8)$ & $172(78.2)$ & 1.00 & 1.00 \\
\hline Male & $120(50.6)$ & $117(49.4)$ & $3.67(2.44-5.53)$ & $3.38(2.22-5.17)$ \\
\hline \multicolumn{5}{|l|}{ Ethnicity } \\
\hline Thai & $28(33.7)$ & $55(66.3)$ & 1.00 & \\
\hline Karen & $140(37.4)$ & $234(62.6)$ & $1.17(0.71-1.94)$ & \\
\hline \multicolumn{5}{|l|}{ Age (years) } \\
\hline $12-17$ years & $67(31.5)$ & $146(68.5)$ & 1.00 & 1.00 \\
\hline $6-11$ years & $101(41.4)$ & $143(58.6)$ & $1.54(1.04-2.26)$ & $2.04(1.31-3.18)$ \\
\hline \multicolumn{5}{|l|}{ Father's education } \\
\hline Educated & $62(33.5)$ & $123(66.5)$ & 1.00 & \\
\hline Uneducated & $106(39.0)$ & $166(61.0)$ & $1.27(0.86-1.87)$ & \\
\hline \multicolumn{5}{|l|}{ Mother's education } \\
\hline Educated & $42(31.3)$ & $92(68.7)$ & 1.00 & \\
\hline Uneducated & $126(39.0)$ & $197(61.0)$ & $1.40(0.91-2.15)$ & \\
\hline \multicolumn{5}{|c|}{ Family income per year (baht) } \\
\hline$\geq 30,000$ & $47(31.1)$ & $104(68.9)$ & 1.00 & \\
\hline$<30,000$ & $121(39.5)$ & $185(60.5)$ & $1.45(0.96-2.19)$ & \\
\hline \multicolumn{5}{|l|}{ Birth weight } \\
\hline$<2500 \mathrm{~g}$ & $44(35.8)$ & 79 (64.2) & 1.00 & \\
\hline$\geq 2500 \mathrm{~g}$ & $117(37.7)$ & $193(62.3)$ & $1.09(0.70-1.68)$ & \\
\hline \multicolumn{5}{|l|}{ Gestational age } \\
\hline$\geq 36$ weeks & $115(36.5)$ & $200(63.5)$ & 1.00 & \\
\hline$<36$ weeks & 47 (39.2) & $73(60.8)$ & $1.12(0.73-1.72)$ & \\
\hline \multicolumn{5}{|l|}{ Parasite infection } \\
\hline Infected & $36(36.7)$ & $62(63.3)$ & 1.00 & \\
\hline None & $39(37.5)$ & $65(62.5)$ & $1.03(0.58-1.83)$ & \\
\hline \multicolumn{5}{|l|}{ School absenteeism } \\
\hline$<1$ week & $129(34.7)$ & $243(65.3)$ & 1.00 & 1.00 \\
\hline$\geq 1$ week & 39 (45.9) & $46(54.1)$ & $1.60(0.99-2.57)$ & $1.72(1.01-2.92)$ \\
\hline
\end{tabular}


Table 5 continued

\begin{tabular}{|c|c|c|c|c|}
\hline \multirow[t]{2}{*}{ Variable } & \multicolumn{2}{|c|}{$\begin{array}{l}\text { School performance in test score of mathematics/ } \\
\text { Thai Language }\end{array}$} & \multirow[t]{2}{*}{ Crude OR } & \multirow[t]{2}{*}{ Adjusted OR } \\
\hline & Poor $(n=168)$ & Not poor $(n=289)$ & & \\
\hline \multicolumn{5}{|l|}{ Emotional intelligence } \\
\hline Normal & $83(30.0)$ & $194(70.0)$ & 1.00 & 1.00 \\
\hline Low & $85(47.2)$ & $95(52.8)$ & $2.09(1.42-3.09)$ & $2.21(1.43-3.41)$ \\
\hline \multicolumn{5}{|l|}{ Nutritional status } \\
\hline \multicolumn{5}{|l|}{ Weight for age } \\
\hline Non poor nutritional status & $146(36.0)$ & $260(64.0)$ & 1.00 & \\
\hline Poor nutritional status & $22(43.1)$ & $29(59.9)$ & $1.35(0.74-2.44)$ & \\
\hline \multicolumn{5}{|l|}{ Height For Age } \\
\hline Non poor nutritional status & $141(36.0)$ & $251(64.0)$ & 1.00 & \\
\hline Poor nutritional status & $27(41.5)$ & $38(58.5)$ & $1.26(0.74-2.16)$ & \\
\hline
\end{tabular}

classroom. Therefore, girls may perform better than boys in class examinations $[28,29]$. The age of students was also associated with school performance in this study. Young children may demonstrate less maturity and lower concentration when learning in comparison with older children.

School performance was also associated with absenteeism. As expected, students who were absent from school for a total of one week or more over a semester had a higher possibility of poor school performance than those who were absent for less than one week. This finding is consistent with that of a previous study conducted in a malaria-endemic area in the Brazilian Amazon [17]. School absenteeism may lead to loss of knowledge provided in the classroom, leading to absent students academically lagging behind other students in the same class.

Low emotional intelligence was associated with poor school performance. The emotional intelligence of children may be affected by many cultural, social, and environmental factors. Emotional intelligence may influence the concentration, attention, and behaviour of children during school, and affect school performance [30-34].

Other potential predictive factors (confounders) for poor school performance were also explored in this study, including socioeconomic status, parents' education, perinatal history, history of intestinal parasitic infection, and current nutritional status. These findings suggested that these factors were not associated with the school performance of students. In this geographic area the population is fairly homogeneous in terms of socioeconomic status, culture, and lifestyle; these factors may therefore not make a difference in the performance of children in school. Although the school participating in this study is the only one in this rural area, it has very good school health programs. Intestinal parasitic infection has been periodically detected and treated. To improve their nutritional status, it is routinely assessed yearly for all students; therefore, chronic malnutrition is rare. Almost $80 \%$ of students in this school had normal nutritional status, which is higher than that reported for other schools in rural areas of Thailand [21]. This fact supports the study results that current nutritional status was not associated with school performance in this population.

However, the cause of poor school performance is multifactorial. Although several potential confounders have been explored and adjusted in this study, some variables may be left unadjusted, such as underlying diseases of students and the learning environment outside school. However, most of the students in the study were healthy; chronic diseases were not observed in this study population. In addition, variation in the learning environment outside school in this rural area is less likely than in urban areas where competition and learning opportunities are greater. This study was conducted in a setting of low malaria transmission where the majority of the population is Karen, a minority group. Therefore, results from this study may not be generalizable to children living in hyperendemic areas or in other urban settings.

The test scores and nutritional status data collected in this study were based on school reports. Although the paper examinations were developed following a standard protocol and a same set of paper examination was given to students in the same grade, the test scores might still vary depending on teachers' assessment. This would affect the reliability of test scores when comparing among different classroom. However, the standardized scores, rather than the raw scores, were used to classified school performance status of students to avoid bias due to variation of grading by different teachers. Errors may also occur when measuring weight and height, especially when performed by different persons or different equipment. However, weight and height data in this school were collected by trained teacher using the same measurement tools; this would ensure the reliability of nutritional status data collected in the study. 
In this study, data on history of malaria infection in students were obtained from the only malaria clinic in the area. The village census record that has been regularly updated was used to identify students who permanently live in the area but did not have malaria infection. Using these data sources helped to ensure the validity of the past malaria status of the subjects. In addition, school performance status was classified using a standardized score to avoid bias that might occur because of variation in standard grading by different teachers in different classrooms. Moreover, this study tracked the history of malaria infection since birth, which provides information on the long-term consequences of uncomplicated malaria in children living in an area of low malaria transmission.

\section{Conclusions}

Childhood malaria infection may affect cognitive function and lead to impairment of memory and language functions. However, the association between childhood malaria infection and school performance was not significance in this study, when the majority of malaria infected children previously had uncomplicated malaria infection in the past three years. These findings suggested that, in low malaria endemic area, the educational consequence after uncomplicated malaria may be less and may not be prolonged.

\section{Authors' contributions}

NV designed and carried out the study, collected data in the field, performed the statistical analysis, interpreted the results, and drafted the manuscript. SL participated in the design of the study and coordination, performed the statistical analysis, interpreted the results, and drafted the manuscript. WP and PJ participated in the design of the study, interpreted the results, and reviewed the manuscript. WM and PR coordinated and participated in data collection. All authors read and approved the final manuscript.

\section{Acknowledgements}

We would like to thank the teachers and students of Rujirapat PrimarySecondary School, Tanousri, Suanphuang, Ratchaburi Province, Thailand and the entire staff of the RTIC for coordinated collection of data. This study was supported by the Mahidol-Oxford Research Unit and the Faculty of Tropical Medicine, Mahidol University and The Thailand Research Fund (TRF).

\section{Competing interests}

The authors declare that they have no competing interests.

Received: 23 March 2015 Accepted: 25 September 2015

Published online: 09 October 2015

\section{References}

1. WHO. World Malaria Report 2014. Geneva, World Health Organization. 2014. Report. http://www.who.int/malaria/world_malaria_report_2014/ en/. Accessed 9 Sep 2015.

2. WHO. World Malaria Report 2012. Geneva, World Health Organization. 2012. Report. http://www.who.int/malaria/publications/world_malaria_ report_2012/en/. Accessed 15 Jan 2015.

3. Nankabirwa J, Brooker SJ, Clarke SE, Fernando D, Gitonga CW, Schellenberg D, Greenwood B. Malaria in school age children in Africa: an increasingly important challenge. Trop Med Int Health. 2014;19:1294-309.
4. Rowe AK, Rowe SY, Snow RW, Korenromp EL, Schellenberg JRA, Stein C, et al. The burden of malaria mortality among African children in the year 2000. Int J Epidemiol. 2006;35:691-704.

5. Meremikwu M, Asindi A, Ezedinachi E. The pattern of neurological sequelae of childhood cerebral malaria among survivors in Calabar, Nigeria. Cent Afr J Med. 1997:43:231-4

6. Boivin MJ. Effects of early cerebral malaria on cognitive ability in Senegalese children. J Dev Behav Pediatr. 2002;23:353-64.

7. Boivin MJ, Bangirana P, Byarugaba J, Opoka RO, Idro R, Jurek AM, et al. Cognitive impairment after cerebral malaria in children: a prospective study. Pediatrics. 2007;119:e360-6.

8. Holding P, Stevenson J, Peshu N, Marsh K. Cognitive sequelae of severe malaria with impaired consciousness. Trans R Soc Trop Med Hyg. 1999;93:529-34

9. Carter J, Mung'ala-Odera V, Neville B, Murira G, Mturi N, Musumba C, et al. Persistent neurocognitive impairments associated with severe falciparum malaria in Kenyan children. J Neurol Neurosurg Psychiatry. 2005;76:476-81.

10. Brabin BJ, Ganley Y. Imported malaria in children in the UK. Arch Dis Child. 1997;77:76-81.

11. Kihara M, Carter JA, Newton CR. The effect of Plasmodium falciparum on cognition: a systematic review. Trop Med Int Health. 2006;11:386-97.

12. Fernando SD, Rodrigo C, Rajapakse S. The 'hidden' burden of malaria: cognitive impairment following infection. Malar J. 2010;9:366.

13. Brown H, Rogerson S, Taylor T, Tembo M, Mwenechanya J, Molyneux $M$, et al. Blood-brain barrier function in cerebral malaria in Malawian children. Am J Trop Med Hyg. 2001;64:207-13.

14. Fernando S, Gunawardena D, Bandara M, De Silva D, Carter R, Mendis K, et al. The impact of repeated malaria attacks on the school performance of children. Am J Trop Med Hyg. 2003;69:582-8.

15. Fernando D, Wickremasinghe R, Mendis K, Wickremasinghe A. Cognitive performance at school entry of children living in malaria-endemic areas of Sri Lanka. Trans R Soc Trop Med Hyg. 2003;97:161-5.

16. Fernando D, De Silva D, Wickremasinghe R. Short-term impact of an acute attack of malaria on the cognitive performance of schoolchildren living in a malaria-endemic area of Sri Lanka. Trans R Soc Trop Med Hyg. 2003;97:633-9.

17. Vitor-Silva S, Reyes-Lecca RC, Pinheiro TR, Lacerda MV. Malaria is associated with poor school performance in an endemic area of the Brazilian Amazon. Malar J. 2009;8:230.

18. Bangirana P, Musisi S, Boivin MJ, Ehnvall A, John CC, Bergemann TL, et al. Malaria with neurological involvement in Ugandan children: effect on cognitive ability, academic achievement and behaviour. Malar J. 2011;10:334.

19. Hay SI, Guerra CA, Tatem AJ, Noor AM, Snow RW. The global distribution and population at risk of malaria: past, present, and future. Lancet Infect Dis. 2004;4:327-36.

20. Malaria disease. http://www.boe.moph.go.th/fact/Malaria.htm. Accessed 2 Feb 2015.

21. Nutritional status of children. http://www.hiso.or.th/hiso/picture/ reportHealth/report/report6_9.pdf. Accessed 2 Jun 2014.

22. Lutter CK, Daelmans BM, de Onis M, Kothari MT, Ruel MT, Arimond M, et al. Undernutrition, poor feeding practices, and low coverage of key nutrition interventions. Pediatrics. 2011;128:e1418-27.

23. The Development of Thai Emotional Intelligence Screening. http://www. klb.dmh.go.th/authenticated.php?m=evaluation\&op=home\&gr=authe nticated_user\&researchld=129. Acccessed 17 Sep 2015.

24. The emotional intelligence for children age 12-17 years old. http://61.19.145.142/tobenumber1cm/images_download/36/36.pdf. Accessed 2 Jun 2014.

25. The emotional intelligence for children age 6-11 years old. http://www. iqeqdekthai.com/survey/Q3Report.php. Accessed 2 Jun 2014

26. Johnson MH. Functional brain development in humans. Nat Rev Neurosci. 2001;2:475-83.

27. Lenroot RK, Giedd JN. Brain development in children and adolescents: insights from anatomical magnetic resonance imaging. Neurosci Biobehav Rev. 2006;30:718-29.

28. McClelland MM, Morrison FJ, Holmes DL. Children at risk for early academic problems: the role of learning-related social skills. Early Child Res Q. 2000;15:307-29. 
29. Bonny AE, Britto MT, Klostermann BK, Hornung RW, Slap GB. School disconnectedness: identifying adolescents at risk. Pediatrics. 2000;106:1017-21.

30. Rousseau C, Drapeau A, Corin E. School performance and emotional problems in refugee children. Am J Orthopsychiatry. 1996;66:239.

31. Newsome S, Day AL, Catano VM. Assessing the predictive validity of emotional intelligence. Pers Individ Differ. 2000;29:1005-16.
32. Gumora G, Arsenio WF. Emotionality, emotion regulation, and school performance in middle school children. J Sch Psychol. 2002;40:395-413.

33. Barchard KA. Does emotional intelligence assist in the prediction of academic success? Educ Psychol Measur. 2003;63:840-58.

34. Graziano PA, Reavis RD, Keane SP, Calkins SD. The role of emotion regulation in children's early academic success. J Sch Psychol. 2007:45:3-19.

Submit your next manuscript to BioMed Central and take full advantage of:

- Convenient online submission

- Thorough peer review

- No space constraints or color figure charges

- Immediate publication on acceptance

- Inclusion in PubMed, CAS, Scopus and Google Scholar

- Research which is freely available for redistribution

Submit your manuscript at

www.biomedcentral.com/submit

(O) Biomed Central 